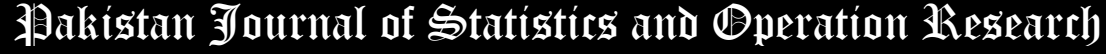

\section{A New Three-parameter Xgamma Fréchet Distribution with Different Methods of Estimation and Applications}

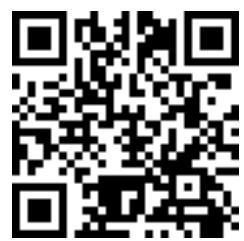

\author{
Mohamed Ibrahim ${ }^{1}$ Laba Handique ${ }^{2}$, Subrata Chakraborty ${ }^{3}$, \\ Nadeem Shafique Butt ${ }^{4}$ and Haitham M. Yousof ${ }^{5 *}$
}

* Corresponding Author

${ }^{1}$ Department of Applied, Mathematical and Actuarial Statistics, Faculty of Commerce, Damietta University, Damietta, Egypt. mohamed_ibrahim@du.edu.eg

${ }^{2}$ Department of Statistics, Dibrugarh University, Assam, India. handiquelaba@gmail.com

${ }^{3}$ Department of Statistics, Dibrugarh University, Assam, India. subrata arya@yahoo.co.in

${ }^{4}$ Department of Family and Community Medicine King Abdul Aziz University, Jeddah,

Kingdom of Saudi Arabia.nshafique@kau.edu.sa

${ }^{5}$ Department of Statistics, Mathematics and Insurance, Benha University, Egypt. haitham.yousof@fcom.bu.edu.eg

\begin{abstract}
In this article an attempt is made to introduce a new extension of the Fréchet model called the Xgamma Fréchet model. Some of its properties are derived. The estimation of the parameters via different estimation methods are discussed. The performances of the proposed estimation methods are investigated through simulations as well as real life data sets. The potentiality of the proposed model is established through modelling of two real life data sets. The results have shown clear preference for the proposed model compared to several know competing ones.
\end{abstract}

Key Words: Xgamma Model; Fréchet Model; Simulations; Least Squares; Cramer-Von-Mises; Maximum Product Spacing Distance; Bootstrapping.

Mathematical Subject Classification: 62N01; 62N02; 62E10.

\section{Introduction and genesis}

The probability density function (PDF) and cumulative distribution function (CDF) of Fréchet (Fr) distribution are given, respectively, by

and

$$
g_{a, b}(x)=\left.b a^{b} x^{-(b+1)} e^{-\left(\frac{a}{x}\right)^{b}}\right|_{x \geq 0}
$$

$$
G_{a, b}(x)=e^{-\left(\frac{a}{x}\right)^{b}}
$$

where $a>0$ is a scale parameter and $b>0$ refers to the shape parameter. Recently, Yousof et al. (2018c) investigated and new family called the extended odd Fréchet family of distributions based on (2). Due to Cordeiro et al. (2020), the CDF of the Xgamma Fréchet (XG-Fr) model can be expressed as

$$
F_{\theta, a, b}(x)=\left.\left[1-\frac{1}{1+\theta}\left[1-e^{-\left(\frac{a}{x}\right)^{b}}\right]^{\theta}\left(\begin{array}{c}
1+\theta-\theta \log \left[1-e^{-\left(\frac{a}{x}\right)^{b}}\right] \\
+\frac{1}{2} \theta^{2}\left\{\log \left[1-e^{-\left(\frac{a}{x}\right)^{b}}\right]\right\}^{2}
\end{array}\right)\right]\right|_{\theta>0 .}
$$

The PDF corresponding to (3) reduces to

$$
f_{\theta, a, b}(x)=\frac{\theta}{1+\theta} b a^{b} x^{-(b+1)} e^{-\left(\frac{a}{x}\right)^{b}}\left[1-e^{-\left(\frac{a}{x}\right)^{b}}\right]^{\theta-1}\left(\theta+\frac{1}{2} \theta^{2}\left\{\log \left[1-e^{-\left(\frac{a}{x}\right)^{b}}\right]\right\}^{2}\right) .
$$

For $a=1$, the XG-Fr reduces to the two-parameter XG-Fr distribution (Yousof et al. (2020)). The XG-Fr density in (4) can be expressed as 


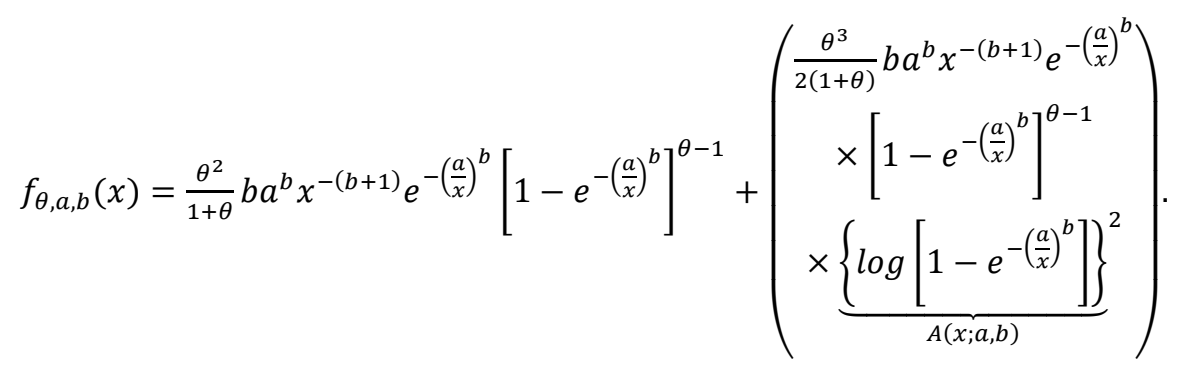

Consider

$$
\log \left(1-\frac{\delta_{1}}{\delta_{2}}\right)=-\left.\sum_{\mathfrak{i}=0}^{\infty}\left(\frac{\delta_{1}}{\delta_{2}}\right)^{\mathfrak{i}+1} \frac{1}{\mathfrak{i}+1}\right|_{\left|\frac{\delta_{1}}{\delta_{2}}\right|_{<1}},
$$

and the power series raised to a positive integer $n$ (Gradshteyn and Ryzhik, 2002, Section 0.314)

$$
\left(\sum_{d=0}^{\infty} a_{d} u^{d}\right)^{n}=\sum_{d=0}^{\infty} c_{(n, d)} u^{d}
$$

where the coefficients $c_{(n, d)}$ (for $d=1,2, \ldots$ ) can be easily determined from the recurrence equation

$$
c_{(n, d)}=\left(d a_{0}\right)^{-1} \sum_{m=1}^{d}[m(n+1)-d] a_{m} c_{(n, d-m)} \quad \text { and } c_{(n, 0)}=a_{0}^{n} .
$$

The coefficient $c_{(n, d)}$ can be calculated from $c_{(n, 0)}, \ldots, c_{(n, d-1)}$ and hence from the quantities $a_{0}, a_{1}, \ldots, a_{d}$. For $\left|\frac{\delta_{1}}{\delta_{2}}\right|<1$ and $\delta_{3}>0$, the power series holds

$$
\left(1-\frac{\delta_{1}}{\delta_{2}}\right)^{\delta_{3}}=\sum_{d=0}^{\infty} \frac{(-1)^{d} \boldsymbol{\Gamma}\left(1+\delta_{3}\right)}{d ! \Gamma\left(1+\delta_{3}-d\right)}\left(\frac{\delta_{1}}{\delta_{2}}\right)^{d} .
$$

Applying (5) to the quantity $A(x ; a, b)$ in the PDF in (4) gives

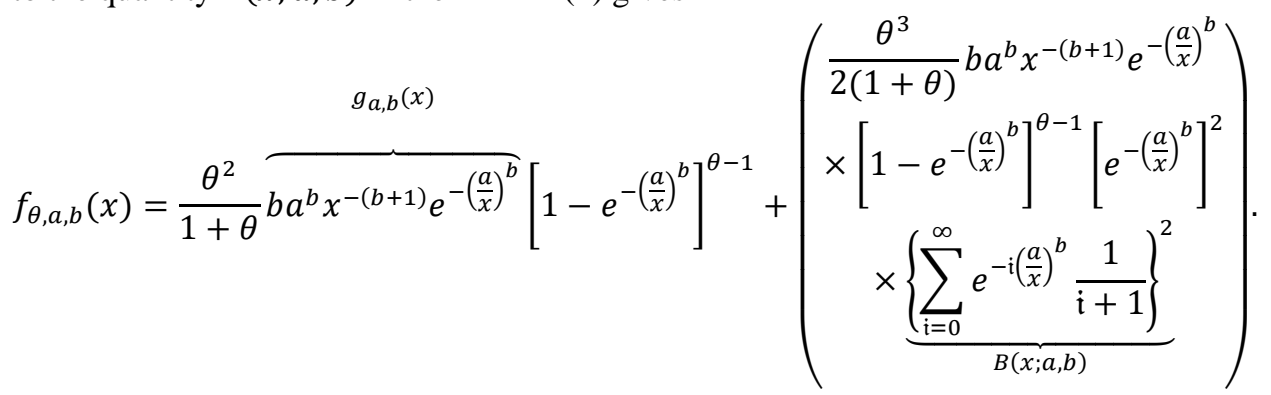

Next, the quantity $B(x ; a, b)$ follows using $(6)$ as

$$
f_{\theta, a, b}(x)=\frac{\theta^{2}}{1+\theta} \overbrace{b a^{b} x^{-(b+1)} e^{-\left(\frac{a}{x}\right)^{b}} \underbrace{g_{a, b}(x)}_{C(x ; a, b, \theta)}}^{\left[1-e^{\left.-\left(\frac{a}{x}\right)^{b}\right]^{\theta-1}}\right.}+\{\times \sum_{i=0}^{\infty} c_{2, i}\left\{e^{-\left(\frac{a}{x}\right)^{b}}\right\}^{2+i} \underbrace{\frac{\theta^{3}}{2(1+\theta)} b a^{b} x^{-(b+1)} e^{-\left(\frac{a}{x}\right)^{b}}}_{c}\}
$$

where $a_{\mathfrak{i}}=1 /(\mathfrak{i}+1)$. Applying the power series (7) to the quantity $C(x ; a, b, \theta)$, we obtain

$$
f_{\theta, a, b}(x)=\sum_{d=0}^{\infty} \mathcal{K}_{d} \pi_{1+d}(x)+\sum_{i=0}^{\infty} \mathcal{K}_{i, d} \pi_{3+i+d}(x),
$$

where

$$
\mathcal{K}_{d}=\theta^{2} \frac{(-1)^{d} \boldsymbol{\Gamma}(\theta)}{(1+d)(1+\theta) \boldsymbol{\Gamma}(\theta-d)}, \quad \mathcal{K}_{\mathrm{i}, d}=\theta^{3} \frac{(-1)^{d} \theta^{3} \boldsymbol{\Gamma}(\theta) c_{2, \mathfrak{i}}}{2(1+\theta)(3+\mathfrak{i}+d) d ! \boldsymbol{\Gamma}(\theta-d)}
$$


and $\pi_{\varphi}(x)$ is the Fr density with scale parameter $a \varphi^{\frac{1}{b}}$ and shape parameter $b$. So, the density of $X$ is a linear combination of Fr densities. The CDF of $X$ follows by integrating (8) as

$$
F_{\theta, a, b}(x)=\sum_{d=0}^{\infty} \mathcal{K}_{d} \Pi_{1+d}(x)+\sum_{i=0}^{\infty} \mathcal{K}_{\mathrm{i}, d} \Pi_{3+i+d}(x),
$$

where $\Pi_{\varphi}(x)$ is the Fr density with scale parameter $a \varphi^{\frac{1}{b}}$ and shape parameter $b$. Equations (8) and (9) are the main results of this section. We provide some plots of the PDF and HRF of the XG-Fr model to show its flexibility. The HRF plots of the XG-Fr distribution can be "upside down" or "monotonically increasing". For more details and useful extension of the Fr model see Harlow (2002), Zaharim et al.(2009), Barreto-Souza et al. (2011), Krishna et al. (2013), Silva et al.(2013), Yousof et al.(2015), Yousof et al. (2016), ul Haq et al. (2017), Yousof et al. (2017a,b,c), Jahanshahi et al. (2019), Korkmaz et al. (2017 and 2018), Yousof et al. (2018a), Chakraborty et al. (2018), Salah et al. (2020)

\section{Properties}

\subsection{Moments}

Let $Y_{\varphi}$ be a rv having density $\pi_{\varphi}(x)$. The $\boldsymbol{r}^{\text {th }}$ ordinary moment of $X$, say $\mu_{r, X}^{\prime}$, follows from (8) as

Then,

$$
\mu_{r, X}^{\prime}=E\left(X^{r}\right)=\sum_{d=0}^{\infty}\left[\mathcal{K}_{d} E\left(Y_{1+d}^{r}\right)+\sum_{i=0}^{\infty} \mathcal{K}_{i, d} E\left(Y_{3+i+d}^{r}\right)\right] .
$$

$$
\mu_{\boldsymbol{r}, X}^{\prime}=E\left(X^{\boldsymbol{r}}\right)=a^{\boldsymbol{r}} \boldsymbol{\Gamma}\left(1-\frac{\boldsymbol{r}}{b}\right) \sum_{d=0}^{\infty}\left[\mathcal{K}_{d}^{(\boldsymbol{r}, 1+d)}+\sum_{\mathrm{i}=0}^{\infty} \mathcal{K}_{\mathrm{i}, d}^{(\boldsymbol{r}, 3+\mathrm{i}+d)}\right] \mathrm{I}_{(b>\boldsymbol{r})},
$$

where

$$
\mathcal{K}_{d}^{(\boldsymbol{r}, 1+d)}=\mathcal{K}_{d}(1+d)^{\frac{r}{b}} \text { and } \mathcal{K}_{\mathrm{i}, d}^{(\boldsymbol{r}, 3+\mathrm{i}+d)}=\mathcal{K}_{\mathrm{i}, d}(3+\mathfrak{i}+d)^{\frac{r}{b}},
$$

and

$$
\left.\Gamma(1+p)\right|_{\left(p \in R^{+}\right)}=p !=\prod_{w=0}^{p-1}(p-w)=\int_{0}^{\infty} e^{-x} x^{p} d x .
$$

Setting $r=1$ in (10) gives the mean of $X$.

\subsection{Incomplete moments}

The $\boldsymbol{r}^{\text {th }}$ incomplete moment of $X$ is defined by

We can write from (8)

$$
m_{r, X}(t)=\int_{-\infty}^{t} x^{r} f_{\theta, a, b}(x) d x
$$

$$
m_{r, X}(t)=\sum_{d=0}^{\infty}\left[\mathcal{K}_{d} m_{r, 1+d}(y)+\sum_{i=0}^{\infty} \mathcal{K}_{\mathrm{i}, d} m_{r, 3+\mathrm{i}+d}(y)\right]
$$

Then,

$$
m_{r, X}(t)=a^{r} \sum_{d=0}^{\infty}\left[\begin{array}{c}
\mathcal{K}_{d}^{(r, 1+d)} \gamma\left(1-\frac{\boldsymbol{r}}{b},(1+d)\left(\frac{a}{t}\right)^{b}\right) \\
+\sum_{\mathrm{i}=0}^{\infty} \mathcal{K}_{\mathrm{i}, d}^{(\boldsymbol{r}, 3+\mathrm{i}+d)} \gamma\left(1-\frac{\boldsymbol{r}}{b},(3+\mathfrak{i}+d)\left(\frac{a}{t}\right)^{b}\right)
\end{array}\right] \mathrm{I}_{(b>\boldsymbol{r})},
$$

where

$$
\left.\gamma(p, q)\right|_{(p \neq 0,-1,-2, \ldots)}=\int_{0}^{q} t^{p-1} e^{-t} d t=\frac{q^{p}}{p}\left\{F_{1,1}[p ; p a+1 ;-q]\right\}=\sum_{d=0}^{\infty} q^{p+d} \frac{(-1)^{d}}{d !(p+d)}=\Gamma(p)-\Gamma(p, q),
$$




$$
\left.\Gamma(p, q)\right|_{(x>0)}=\int_{q}^{\infty} e^{-t} t^{p-1} d t
$$

and $F_{1,1}[\because, \cdot$,$] is a confluent hypergeometric function (see Johnson et al. (2005)). Setting r=1$ in (11) gives the first incomplete moment

$$
m_{1, X}(t)=\left.a \sum_{d=0}^{\infty}\left[\begin{array}{c}
\mathcal{K}_{d}^{(1,1+d)} \gamma\left(1-\frac{1}{b},(1+d)\left(\frac{a}{t}\right)^{b}\right) \\
+\sum_{i=0}^{\infty} \mathcal{K}_{i, d}^{(1,3+i+d)} \gamma\left(1-\frac{1}{b},(3+\mathfrak{i}+d)\left(\frac{a}{t}\right)^{b}\right)
\end{array}\right]\right|_{(b>1)}
$$

Two important applications of the $m_{1, X}(t)$ are related to the mean deviation about the mean $\left(\delta_{1, X}\right)$ and median and to the Bonferroni and Lorenz curves. The mean deviation about the mean

and about the median

$$
\delta_{1, X}=E(|X-E(X)|)=2 \mu_{1, X}^{\prime} F(E(X))-2 m_{1}(E(X))
$$

$$
\delta_{2, X}=E(|X-M|)=E(X)-2 m_{1, X}(M)
$$

where $E(X), M=Q(u)=F^{-1}(u)$ is the median of $X, F\left(\mu_{1, X}^{\prime}\right)$ is easily calculated from (3).

\subsection{Moment generating function}

The moment generating function (MGF) of $X$, say $M_{X}(t)=E\left(e^{t X}\right)$, is obtained from (8) as

$$
M_{X}(t)=\sum_{d=0}^{\infty}\left[\mathcal{K}_{d} M_{1+d}(t)+\sum_{\mathrm{i}=0}^{\infty} \mathcal{K}_{\mathrm{i}, d} M_{3+\mathrm{i}+d}(t)\right] \text {. }
$$

Then,

$$
M_{X}(t)=\left.\sum_{d, \boldsymbol{r}=0}^{\infty}\left[\begin{array}{c}
\mathcal{K}_{d}^{(\boldsymbol{r}, 1+d)}\left[(t a)^{\boldsymbol{r}} / \boldsymbol{r} !\right] \Gamma\left(1-\frac{\boldsymbol{r}}{b}\right) \\
\sum_{i=0}^{\infty} \mathcal{K}_{i, d}^{(\boldsymbol{r}, 3+i+d)}\left[(t a)^{\boldsymbol{r}} / \boldsymbol{r} !\right] \Gamma\left(1-\frac{\boldsymbol{r}}{b}\right)
\end{array}\right]\right|_{(b>\boldsymbol{r})},
$$

where $M_{\varphi}(t)$ is the generating function of $Y_{\varphi}$. The last two integrals can be computed numerically for most parent distributions. Consider the Wright generalized hypergeometric function (see Wright (1935)) defined by

Then, we can write $M(t)$ as

$$
\boldsymbol{\Psi}_{p, q}\left[\begin{array}{l}
a_{1}, A_{1}, \ldots, a_{p}, A_{p} \\
\left.b_{1}, B_{1}, \ldots, b_{q}, B_{q} ; x\right]=\sum_{n=0}^{\infty} \frac{\prod_{d=1}^{p} \quad \Gamma\left(a_{d}+A_{d} n\right)}{\prod_{d=1}^{q}} \frac{x^{n}}{n !}\left(b_{d}+B_{d} n\right)
\end{array}\right.
$$

$$
M(t)=\Psi_{1,0}\left[\left(1,-\frac{1}{b}\right) ; a t\right] .
$$

Combining expressions (8) and (12), we obtain the MGF of $X$, say $M_{X}(t)$, as

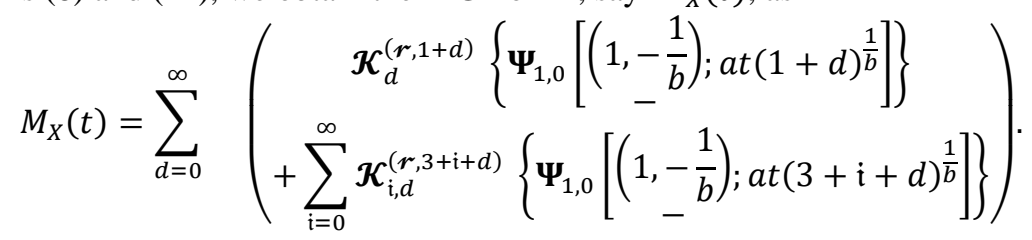

Numerical analysis for the $\mathrm{E}(\mathrm{X})$, variance $(\operatorname{Var}(\mathrm{X})$ ). skewness $($ Ske $(\mathrm{X}))$ and kurtosis $(\mathrm{Ku}(\mathrm{X}))$ measures. In this section we explore the some commonly used descriptive measure numerically. Based on table 1, we note that:

I- $\quad$ The value of $E(X)$ of the new model decreases as $b$ and $\theta$ increases.

II- The value of $E(X)$ of the new model increases as $a$ increases.

III- The skewness of the new model can be only positive in the interval $(0.00004,31.43)$.

IV- The parameter $a$ has a very little effect on $\operatorname{Ske}(X))$ and $\mathrm{Ku}(X)$.

V- The $\mathrm{Ku}(X)$ of new model can be only more than 3 .

Table 1: $\mathrm{E}(\mathrm{X}), \operatorname{Var}(\mathrm{X}), \mathrm{Ske}(\mathrm{X})$ and $\mathrm{Ku}(\mathrm{X})$ of the $\mathrm{XG}-\mathrm{Fr}$ distribution.

\begin{tabular}{ccccccc}
\hline$\theta$ & $\mathrm{a}$ & $\mathrm{b}$ & $\mathrm{E}(\mathrm{X})$ & $\mathrm{Var}(\mathrm{X})$ & $\mathrm{Ske}(\mathrm{X})$ & $\mathrm{Ku}(\mathrm{X})$ \\
\hline 2 & 2 & 2.5 & 2.58199 & 2.23623 & 17.72601 & 83.0921 \\
& & 3 & 2.43245 & 1.21732 & 9.00210 & 28.8696
\end{tabular}




\begin{tabular}{ccccccc} 
& & 3.5 & 2.34159 & 0.76736 & 5.92687 & 17.5192 \\
& & 5 & 2.20628 & 0.29634 & 2.98242 & 9.15719 \\
& & 6 & 2.16219 & 0.18979 & 2.30250 & 7.52120 \\
& & & & & & \\
1.5 & 1.5 & 3 & 2.23562 & 2.008860 & 31.42609 & 314.195 \\
& 5 & & 7.45207 & 22.32067 & 31.42603 & 314.317 \\
& 10 & & 14.90414 & 89.28269 & 31.42605 & 314.274 \\
& 20 & & 29.80828 & 357.1308 & 31.42605 & 314.285 \\
& 25 & & 37.26035 & 558.0165 & 31.42614 & 314.543 \\
& & & & & & \\
1.5 & 4 & 4 & 5.25901 & 5.02084 & 9.34309 & 30.0971 \\
2 & & & 4.56222 & 2.11634 & 4.42232 & 12.9739 \\
10 & & & 3.18855 & 0.13175 & 0.31359 & 3.62146 \\
50 & & & 2.78359 & 0.03562 & 0.00004 & 3.00655 \\
100 & & & 2.67578 & 0.02428 & 0.02060 & 3.01222 \\
\hline
\end{tabular}

\section{Estimation}

\subsection{Maximum likelihood estimation (MLE)}

Here, we consider the estimation of the unknown parameters of the new family from complete samples by maximum likelihood. Let $x_{1}, x_{2}, \cdots, x_{n}$ be a random sample from the XG-Fr model. Then, the log-likelihood function for $\boldsymbol{\Phi}$ is given by

$$
\begin{aligned}
\ell_{n}(\boldsymbol{\Phi}) & =n \log \theta-n \log (1+\theta)+n \log b+n b \log a-(b+1) \sum_{\mathfrak{i}=1}^{n} \log x_{\mathfrak{i}} \\
& -\sum_{i=1}^{n}\left(\frac{a}{x_{\mathfrak{i}}}\right)^{b}+(\theta-1) \sum_{i=1}^{n} \log p_{\mathfrak{i}}+\sum_{i=1}^{n} \log \left[\theta+\frac{1}{2} \theta^{2}\left(\log p_{\mathfrak{i}}\right)^{2}\right],
\end{aligned}
$$

where

$$
p_{\mathrm{i}}=1-e^{-\left(\frac{a}{x_{\mathrm{i}}}\right)^{b}}
$$

The log-likelihood function in (13) can be maximized numerically by using R (optim), SAS (PROC NLMIXED) or Ox program (sub-routine MaxBFGS), among others. For interval estimation of the parameters, the elements of the $3 \times 3$ observed information matrix $\mathbf{J}(\boldsymbol{\Phi})$ can be evaluated numerically.

\subsection{Ordinary and weighted least-squares estimators}

The theory of least square estimation and weighted least square estimation was proposed by Swain et al. (1988) to estimate the parameters of the Beta distribution. It is based on the minimization of the sum of the square of differences of theoretical cumulative distribution function and empirical distribution function. Suppose $F_{\theta, a, b}\left(x_{\mathfrak{i}}\right)$ denotes the distribution function of XG-Fr distribution and if $x_{1}<x_{2}<\cdots<x_{n}$ be the $n$ ordered random sample. The ordinary least square estimates (OLSEs) are obtained by minimizing

Now using (3) we have

$$
O L S(\theta, a, b)=\sum_{\mathfrak{i}=1}^{n}\left[F_{\theta, a, b}\left(x_{\mathfrak{i}}\right)-\frac{\mathfrak{i}}{n+1}\right]^{2} .
$$

$$
O L S(\theta, a, b)=\sum_{i=1}^{n}\left\{\left[1-\frac{\left[1-e^{-\left(\frac{a}{x_{i}}\right)^{b}}\right]^{\theta}}{1+\theta}\left(\begin{array}{c}
\left.\left.1+\theta-\theta \log \left[1-e^{-\left(\frac{a}{x_{i}}\right)^{b}}\right]\right)\right]-\frac{\mathfrak{i}}{n+1} \\
+\frac{1}{2} \theta^{2}\left\{\log \left[1-e^{\left.-\left(\frac{a}{x_{i}}\right)^{b}\right]}\right\}^{2}\right.
\end{array}\right)\right]\right.
$$

The, least square estimators (LSE) of the parameters are obtained by simultaneously solving the following nonlinear equations: 


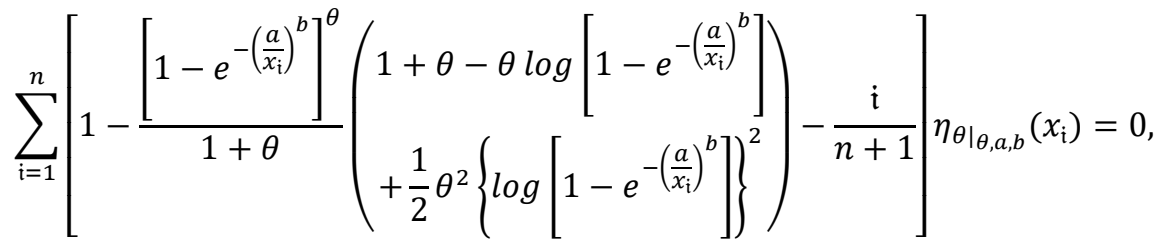

$$
\begin{aligned}
& \sum_{\mathfrak{i}=1}^{n}\left[1-\frac{\left[1-e^{-\left(\frac{a}{x_{\mathrm{i}}}\right)^{b}}\right]^{\theta}}{1+\theta}\left(\begin{array}{c}
1+\theta-\theta \log \left[1-e^{-\left(\frac{a}{x_{\mathrm{i}}}\right)^{b}}\right] \\
+\frac{1}{2} \theta^{2}\left\{\log \left[1-e^{\left.-\left(\frac{a}{x_{\mathrm{i}}}\right)^{b}\right]}\right]\right\}
\end{array}\right]-\frac{\mathfrak{i}}{n+1}\right] \eta_{\left.a\right|_{\theta, a, b}}\left(x_{\mathfrak{i}}\right)=0
\end{aligned}
$$

and

$$
\sum_{\mathfrak{i}=1}^{n}\left[1-\frac{\left[1-e^{-\left(\frac{a}{x_{i}}\right)^{b}}\right]^{\theta}}{1+\theta}\left(\begin{array}{c}
1+\theta-\theta \log \left[1-e^{-\left(\frac{a}{x_{\mathfrak{i}}}\right)^{b}}\right] \\
+\frac{1}{2} \theta^{2}\left\{\log \left[1-e^{\left.-\left(\frac{a}{x_{\mathrm{i}}}\right)^{b}\right]}\right]\right.
\end{array}\right]-\frac{\mathfrak{i}}{n+1}\right] \eta_{\left.b\right|_{\theta, a, b}\left(x_{\mathfrak{i}}\right)=0}
$$

where $\eta_{\left.\theta\right|_{\theta, a, b}}\left(x_{\mathfrak{i}}\right), \quad \eta_{\left.a\right|_{\theta, a, b}}\left(x_{\mathfrak{i}}\right)$ and $\eta_{\left.b\right|_{\theta, a, b}}\left(x_{\mathfrak{i}}\right)$ are the values of the first derivatives with respect to (w.r.t.) parameters of XG-Fr distribution. The weighted least squares estimates (WLSE) are obtained by minimizing the given form of equation with respect to the parameters

$$
W L S(\theta, a, b)=\sum_{\mathfrak{i}=1}^{n} \frac{(n+1)^{2}(n+2)}{\mathfrak{i}(n-\mathfrak{i}+1)}\left[F_{\theta, a, b}\left(x_{\mathfrak{i}}\right)-\frac{\mathfrak{i}}{n+1}\right]^{2} .
$$

The WLSE of the parameters are obtained by solving the following non-linear equations:

$$
\begin{aligned}
& \sum_{\mathfrak{i}=1}^{n} \frac{(n+1)^{2}(n+2)}{\mathfrak{i}(n-\mathfrak{i}+1)}\left[1-\frac{\left[1-e^{-\left(\frac{a}{x_{\mathfrak{i}}}\right)^{b}}\right]^{\theta}}{1+\theta}\left(\begin{array}{c}
1+\theta-\theta \log \left[1-e^{\left.-\left(\frac{a}{x_{\mathfrak{i}}}\right)^{b}\right]}\right. \\
+\frac{1}{2} \theta^{2}\left\{\log \left[1-e^{\left.\left.-\left(\frac{a}{x_{\mathfrak{i}}}\right)^{b}\right]\right\}^{2}}\right]\right. \\
-\frac{\mathfrak{i}}{n+1}
\end{array}\right] \eta_{\left.\theta\right|_{\theta, a, b}\left(x_{\mathfrak{i}}\right)=0,}\right. \\
& \sum_{\mathfrak{i}=1}^{n} \frac{(n+1)^{2}(n+2)}{\mathfrak{i}(n-\mathfrak{i}+1)}\left[1-\frac{\left[1-e^{-\left(\frac{a}{x_{\mathfrak{i}}}\right)^{b}}\right]^{\theta}}{1+\theta}\left[\begin{array}{c}
\left.1+\theta-\theta \log \left[1-e^{-\left(\frac{a}{x_{\mathfrak{i}}}\right)^{b}}\right]\right) \\
+\frac{1}{2} \theta^{2}\left\{\log \left[1-e^{\left.-\left(\frac{a}{x_{\mathfrak{i}}}\right)^{b}\right]}\right]\right\}^{2} \\
-\frac{\mathfrak{i}}{n+1}
\end{array}\right] \eta_{\left.a\right|_{\theta, a, b}\left(x_{\mathfrak{i}}\right)=0,}\right.
\end{aligned}
$$

and 


$$
\sum_{\mathfrak{i}=1}^{n} \frac{(n+1)^{2}(n+2)}{\mathfrak{i}(n-\mathfrak{i}+1)}\left[1-\frac{\left[1-e^{-\left(\frac{a}{x_{\mathfrak{i}}}\right)^{b}}\right]^{\theta}}{1+\theta}\left[\begin{array}{c}
1+\theta-\theta \log \left[1-e^{-\left(\frac{a}{x_{\mathfrak{i}}}\right)^{b}}\right] \\
+\frac{1}{2} \theta^{2}\left\{\log \left[1-e^{\left.-\left(\frac{a}{x_{\mathfrak{i}}}\right)^{b}\right]}\right]\right\}^{2} \\
-\frac{\mathfrak{i}}{n+1}
\end{array}\right] \eta_{\left.b\right|_{\theta, a, b}\left(x_{\mathfrak{i}}\right)=0,}\right.
$$

where, $\eta_{\left.\theta\right|_{\theta, a, b}}\left(x_{\mathfrak{i}}\right), \quad \eta_{\left.a\right|_{\theta, a, b}}\left(x_{\mathfrak{i}}\right)$ and $\eta_{\left.b\right|_{\theta, a, b}}\left(x_{\mathfrak{i}}\right)$ are the values of first derivatives of the CDF of XG-Fr distribution.

\subsection{Method of Cramer-Von-Mises estimation}

The Cramer-Von- Mises estimation (CVME) method of the parameters is based on the theory of minimum distance estimation. It was proposed and justified that the bias of the estimator is smaller than the other minimum distance estimators. Thus, The Crammer-Von Mises estimates of the parameter $\theta, a, b$ are obtained by minimizing the following expression w.r.t. to the parameters $\theta, a, b$ respectively.

and

$$
\operatorname{CVM}(\theta, a, b)=\frac{1}{12 n}+\sum_{\mathfrak{i}=1}^{n}\left[F_{\theta, a, b}\left(x_{\mathfrak{i}}\right)-\frac{2 \mathfrak{i}-1}{2 n}\right]^{2},
$$

$$
\operatorname{CVM}(\theta, a, b)=\sum_{\mathfrak{i}=1}^{n}\left[1-\frac{\left[1-e^{-\left(\frac{a}{x_{\mathrm{i}}}\right)^{b}}\right]^{\theta}}{1+\theta}\left(\begin{array}{c}
1+\theta-\theta \log \left[1-e^{-\left(\frac{a}{x_{\mathrm{i}}}\right)^{b}}\right] \\
+\frac{1}{2} \theta^{2}\left\{\log \left[1-e^{\left.-\left(\frac{a}{x_{\mathrm{i}}}\right)^{b}\right]}\right\}^{2}\right.
\end{array}\right]^{2}-\frac{2 \mathfrak{i}-1}{2 n}\right]^{2}
$$

The CVME of the parameters are obtained by solving the following non-linear equations

$$
\begin{aligned}
& \sum_{i=1}^{n}\left[1-\frac{\left[1-e^{-\left(\frac{a}{x_{i}}\right)^{b}}\right]^{\theta}}{1+\theta}\left(\begin{array}{c}
1+\theta-\theta \log \left[1-e^{\left.-\left(\frac{a}{x_{i}}\right)^{b}\right]}\right. \\
+\frac{1}{2} \theta^{2}\left\{\log \left[1-e^{\left.-\left(\frac{a}{x_{\mathfrak{i}}}\right)^{b}\right]}\right]\right\}
\end{array}\right]-\frac{2 \mathfrak{i}-1}{2 n}\right] \eta_{\left.\theta\right|_{\theta, a, b}}\left(x_{\mathfrak{i}}\right)=0, \\
& \sum_{i=1}^{n}\left[1-\frac{\left[1-e^{-\left(\frac{a}{x_{\mathrm{i}}}\right)^{b}}\right]^{\theta}}{1+\theta}\left(\begin{array}{c}
1+\theta-\theta \log \left[1-e^{\left.-\left(\frac{a}{x_{\mathrm{i}}}\right)^{b}\right]}\right. \\
+\frac{1}{2} \theta^{2}\left\{\log \left[1-e^{\left.-\left(\frac{a}{x_{\mathrm{i}}}\right)^{b}\right]}\right]\right\}
\end{array}\right]-\frac{2 \mathfrak{i}-1}{2 n}\right] \eta_{\left.a\right|_{\theta, a, b}}\left(x_{\mathfrak{i}}\right)=0
\end{aligned}
$$

and

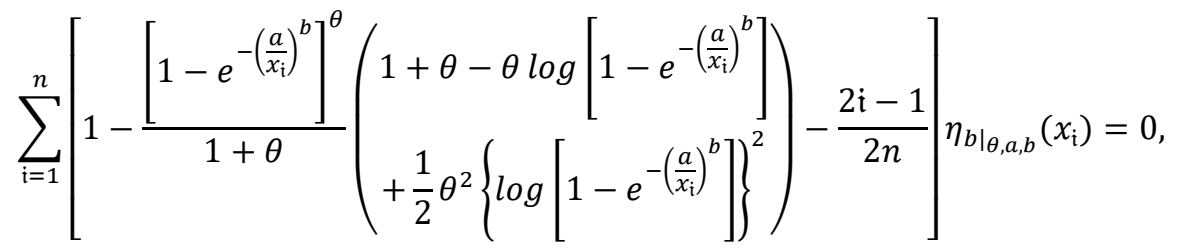

where $\eta_{\left.\theta\right|_{\theta, a, b}}\left(x_{\mathfrak{i}}\right), \quad \eta_{\left.a\right|_{\theta, a, b}}\left(x_{\mathfrak{i}}\right)$ and $\eta_{\left.b\right|_{\theta, a, b}}\left(x_{\mathfrak{i}}\right)$ are the values of the first derivatives of the CDF of XG-Fr distribution with respect to $\theta, a, b$ respectively.

\subsection{Maximum product spacing distance estimators}


The maximum product of spacings distance (MPSD) method has been originally proposed by Cheng and Amin (1983) as an alternative to MLE for the estimation of parameters of continuous univariate models. Let $\left.\operatorname{MPS} D_{\mathfrak{i}}\right|_{\theta, a, b}\left(x_{\mathfrak{i}}: n\right)$ be the uniform spacings of a random sample from the XG-Fr model where

$$
\begin{gathered}
M P S D_{\left.\mathfrak{i}\right|_{\theta, a, b}}\left(x_{\mathfrak{i}}: n\right)=F_{\theta, a, b}\left(x_{\mathfrak{i}: n}\right)-F_{\theta, a, b}\left(x_{\mathfrak{i}-1: n}\right), \\
F_{\theta, a, b}\left(x_{\mathfrak{i}=0: n}\right)=0, F_{\theta, a, b}\left(x_{\mathfrak{i}=n+1: n}\right)=1 \text { and } \sum_{\mathfrak{i}=1}^{n+1} M P S D_{\left.\mathfrak{i}\right|_{\theta, a, b}}\left(x_{\mathfrak{i}: n}\right)=1,
\end{gathered}
$$

then, the maximum product of spacings estimators of the unknown parameters $\theta, a$ and $b$ will be obtained by maximizing the geometric mean of the spacings with respect to $\theta, a$ and $b$, where

$$
\operatorname{Geom}_{\theta, a, b}\left(\operatorname{MPS}_{\left.\mathfrak{i}\right|_{\theta, a, b}}\left(x_{\mathfrak{i}}: n\right)\right)=\left[\prod_{\mathfrak{i}=1}^{1+n} \operatorname{MPSD}_{\left.\mathfrak{i}\right|_{\theta, a, b}}\left(x_{\mathfrak{i}}: n\right)\right]^{\frac{1}{1+n}}
$$

or by maximizing the function

$$
H_{\theta, a, b}\left(\operatorname{MPS} D_{\left.\mathfrak{i}\right|_{\theta, a, b}}\left(x_{\mathfrak{i}}: n\right)\right)=\frac{1}{1+n} \sum_{\mathfrak{i}=1}^{n+1} \log \operatorname{MPS} D_{\left.\mathfrak{i}\right|_{\theta, a, b}}\left(x_{\mathfrak{i}: n}\right),
$$

the estimators of the unknown parameters $\theta, a$ and $b$ can also be obtained by solving the nonlinear equations where

$$
\frac{\partial}{\partial \theta} H_{\theta, a, b}\left(\operatorname{MPSD}_{\left.\mathfrak{i}\right|_{\theta, a, b}}\left(x_{\mathfrak{i}}: n\right)\right)=\frac{\partial}{\partial a} H_{\theta, a, b}\left(\operatorname{MPSD}_{\left.\mathfrak{i}\right|_{\theta, a, b}}\left(x_{\mathfrak{i}}: n\right)\right)=\frac{\partial}{\partial b} H_{\theta, a, b}\left(\operatorname{MPS} D_{\left.\mathfrak{i}\right|_{\theta, a, b}}\left(x_{\mathfrak{i}}: n\right)\right)=0,
$$

$$
\begin{aligned}
\frac{\partial}{\partial \theta} H_{\theta, a, b}\left(\operatorname{MPSD}_{\left.\mathfrak{i}\right|_{\theta, a, b}}\left(x_{\mathfrak{i}: n}\right)\right) & =\sum_{i=1}^{n+1} \frac{\eta_{\left.\theta\right|_{\theta, a, b}}\left(x_{\mathfrak{i}: n}\right)-\eta_{\left.\theta\right|_{\theta, a, b}}\left(x_{\mathfrak{i}-1: n}\right)}{M P S D_{\left.\mathfrak{i}\right|_{\theta, a, b}}\left(x_{\mathfrak{i}: n}\right)}, \\
\frac{\partial}{\partial a} H_{\theta, a, b}\left(\operatorname{MPSD}_{\left.\mathfrak{i}\right|_{\theta, a, b}}\left(x_{\mathfrak{i}: n}\right)\right) & =\sum_{i=1}^{n+1} \frac{\eta_{\left.a\right|_{\theta, a, b}}\left(x_{\mathfrak{i}: n}\right)-\eta_{\left.a\right|_{\theta, a, b}}\left(x_{\mathfrak{i}-1: n}\right)}{\operatorname{MPSD}_{\left.\mathfrak{i}\right|_{\theta, a, b}}\left(x_{\mathfrak{i}: n}\right)},
\end{aligned}
$$

and

where $\eta_{\left.\cdot\right|_{\theta, a, b}}(x$.$) is defined before.$

$$
\frac{\partial}{\partial b} H_{\theta, a, b}\left(\operatorname{MPSD}_{\left.\mathfrak{i}\right|_{\theta, a, b}}\left(x_{\mathfrak{i}: n}\right)\right)=\sum_{i=1}^{n+1} \frac{\eta_{\left.b\right|_{\theta, a, b}}\left(x_{\mathfrak{i}: n}\right)-\eta_{\left.b\right|_{\theta, a, b}}\left(x_{\mathfrak{i}-1: n}\right)}{\operatorname{MPSD}_{\left.\mathfrak{i}\right|_{\theta, a, b}}\left(x_{\mathfrak{i}: n}\right)}
$$

\subsection{Bootstrapping method}

Bootstrapping method is a powerful statistical technique. It is especially useful when the sample size that we are working with is small. Under the usual circumstances, sample sizes of less than 40 cannot be dealt with by assuming a normal or a $t$ distributions. Bootstrap techniques work quite well with samples that have less than 40 elements. The reason for this is that bootstrapping involves resampling. These kinds of techniques assume nothing about the distribution of our data. Bootstrapping has become more popular as computing resources have become more readily available. This is because for bootstrapping to be practical a computer must be used. We will see how this works in the following Sections.

\section{Comparing fits}

We shall compare the fits of the XG-Fr distribution with those of other competitive models, namely: Fréchet (Fr), Marshal-Olkin Fréchet (MO-Fr), Generalized Marshal-Olkin Fréchet (GMO-Fr), Kumaraswamy Fréchet (Kw-Fr), beta Fréchet (B-Fr), Kumaraswamy Marshal-Olkin Fréchet (KwMO-Fr), Marshal-Olkin Kumaraswamy Fréchet (MOKw-Fr). Data I: This data set (gauge lengths of $20 \mathrm{~mm}$ ) (see Kundu and Raqab, 2009) consists of 74 observations $\{1.312,1.314,1.479,1.552,1.700,1.803,1.861,1.865,1.944,1.958,1.966,1.997,2.006,2.021,2.027,2.055,2.063$, $2.098,2.140,2.179,2.224,2.240,2.253,2.270,2.272,2.274,2.301,2.301,2.359,2.382,2.382,2.426,2.434,2.435$, $2.478,2.490,2.511,2.514,2.535,2.554,2.566,2.570,2.586,2.629,2.633,2.642,2.648,2.684,2.697,2.726,2.770$, 
2.773, 2.800, 2.809, 2.818, 2.821, 2.848, 2.880, 2.809, 2.818, 2.821, 2.848, 2.880, 2.954, 3.012, 3.067, 3.084, 3.090, $3.096,3.128,3.233,3.433,3.585,3.585\}$. Data II: This data set (failure times (in days) of 72 guinea pigs infected with virulent tubercle bacilli, observed and reported by Bjerkedal (1960) $\{10,33,44,56,59,72,74,77,92,93,96,100$, 100, 102, 105, 107, 107,108, 108, 108, 109, 112, 113, 115, 116, 120, 121, 122, 122, 124, 130, 134, 136, 139, 144, $146,153,159,160,163,163,168,171,172,176,183,195,196,197,202,213,215,216,222,230,231,240,245$, $251,253,254,255,278,293,327,342,347,361,402,432,458,555\}$. Many other real-life data sets can be analyzed for comparing the competitive Fréchet extensions see Aryal and Yousof (2017), Aryal et al. (2017), Brito et al. (2017), Cordeiro et al. (2018), Yousof et al. (2018a and 2019), Gad et al. (2019), Yadav et al. (2019), Lak et al. (2019), Elsayed and Yousof (2019a,b and 2020)Al-babtain et al. (2020b), Alizadeh et al. (2020), Karamikabir et al. (2020). Figure 1 gives the total time test (TTT) plot (see Aarset (1987)) for the two data sets. The TTT is an important graphical approach to verify whether the data can be applied to a specific distribution or not. Aarset (1987) showed that the HRF is constant if the TTT plot is graphically presented as a straight diagonal, the HRF is increasing (or decreasing) if the TTT plot is concave (or convex). The HRF is U-shaped (bathtub) if the TTT plot is firstly convex and then concave, if not, the HRF is unimodal. This plot indicates that the empirical HRFs of the two data sets are "monotonically increasing".
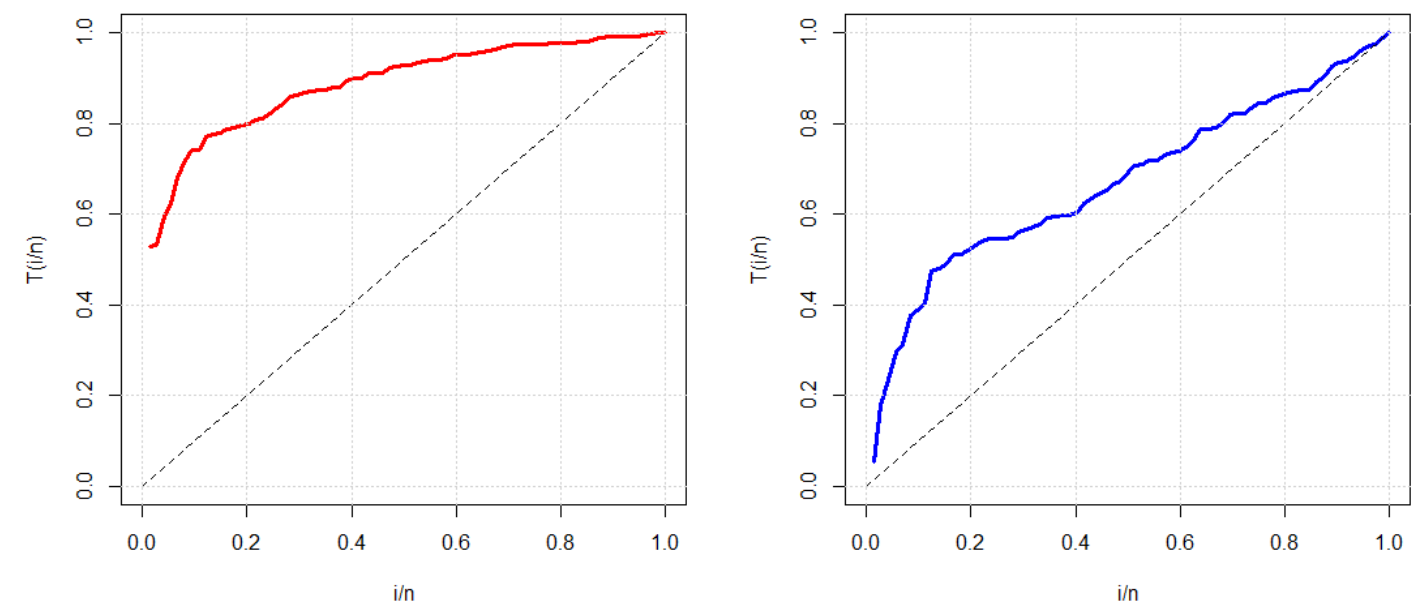

Figure 1: The TTT plots of data I and II.

We consider the Cramér-Von Mises and the Anderson-Darling $\left(W^{*}, A^{*}\right)$ and the Kolmogorov-Smirnov (KS) statistic. Moreover, we consider some other goodness-of-fit measures including the Akaike information criterion $(A I C)$, consistent Akaike information criterion ( CAIC ), Hannan-Quinn information criterion (HQIC), Bayesian information criterion $(B I C)$. The numerical values of the model selection statistics are listed in Tables 2 and 4 . Tables 3 and 5 list the MLEs and their corresponding standard errors (SEs) (in parentheses) as well as the confidence intervals (CIs) in [square brackets] of the model parameters. We proved empirically that the XG-Fr distribution provides better fits to two real data sets than other seven extended Fr distributions (see Table 3 and Table 5), that results is supported by Figure 2 and Figure 3.

Table 2: MLEs, standard errors, confidence intervals (in parentheses) values for the data set I.

\begin{tabular}{cc}
\hline Models & Estimates \\
\hline $\operatorname{Fr}(\mathrm{a}, \mathrm{b})$ & $4.110,2.169$ \\
& $(0.323),(0.065)$
\end{tabular}

[3.48,4.74], [2.04,2.29]

$\operatorname{MO}-\operatorname{Fr}(\alpha, a, b)$

$80.338,8.0311 .419$ 
GMO-Fr( $\theta, \alpha, a, b)$

$\mathrm{Kw}-\mathrm{Fr}(\beta, \delta, \mathrm{a}, \mathrm{b})$

$\mathrm{B}-\operatorname{Fr}(\beta, \delta, \mathrm{a}, \mathrm{b})$

$\operatorname{KwMO}-\operatorname{Fr}(\alpha, \beta, \delta, \mathrm{a}, \mathrm{b})$

$\operatorname{MOKw}-\operatorname{Fr}(\alpha, \beta, \delta, \mathrm{a}, \mathrm{b})$

$\mathrm{XG}-\operatorname{Fr}(\theta, \mathrm{a}, \mathrm{b})$
(62.007), (0.764), (0.109)

[0,201.87], [6.53,9.53], [1.21,1.63]

3.702, 63.707, 5.918, 1.577

(2.683), (38.655), (0.945), (0.139)

[0,8.96], [0,139.47], [4.06,7.77], [1.30,1.85]

$3.218,217.031,1.005,4.384$

(1.036), (268.565), (0.223), (1.012)

[1.18,5.25], [0,743.42], [0.57,1.44], [2.40,6.37]

2.039, 5.857, 0.242, 37.179

(1.015), (1.813), (0.377), (33.510)

[0.04,4.02], [2.30,9.41], [0,0.98], [0,102.85]

$0.016,0.827,16.985,0.894,25.127$

(0.023), (0.789), (24.975), (0.396), 19.688)

[0,0.06], [0,2.37], [0,65.93], [0.11,1.67], [0,63.72]

$7.995,2.933,35.707,1.221,2.415$

(13.063), (0.825), (41.500), (0.412), (1.032)

[0,33.59], [1.31,4.55], [0,117.05], [0.41,2.03], [0.39,4.44]

$78.511,8.662,1.229$

(71.451), (3.497), (0.242)

$[0,218.55],[1.81,15.52],[0.75,1.70]$

Table 3: Log-likelihood, AIC, BIC, CAIC, HQIC, A, W and KS ( $p$-value) values for data set I.

\begin{tabular}{ccc}
\hline Models & AIC, BIC, CAIC, HQIC & A $^{*}, \mathrm{~W}^{*}, \mathrm{~K} . \mathrm{S} .$, and $(\mathrm{p}$-value) \\
\hline Fr & $142.02,146.63,142.19,143.86$ & $2.93,0.46,0.15,(0.09)$ \\
MO-Fr & $115.06,121.96,115.40,117.81$ & $0.78,0.12,0.07,(0.86)$ \\
GMO-Fr & $112.80,122.00,113.37,126.48$ & $0.39,0.07,0.06,(0.94)$ \\
Kw-Fr & $113.68,122.82,114.25,117.36$ & $0.37,0.07,0.05 .(0.92)$ \\
B-Fr & $112.63,121.84,113.21,116.30$ & $0.44,0.07,0.06,(0.93)$ \\
KwMO-Fr & $113.30,124.82,114.18,117.90$ & $0.34,0.05,0.06,(0.95)$ \\
MOKw-Fr & $113.19,124.68,114.07,117.78$ & $0.35,0.05,0.05,(0.94)$ \\
XG-Fr & $108.66,115.56,109.00,111.42$ & $0.29,0.04,0.05,(0.98)$ \\
\hline
\end{tabular}

Table 2: MLEs, standard errors, confidence intervals (in parentheses) values for the data set II.

\begin{tabular}{cc}
\hline Models & Estimates \\
\hline Fr(a,b) & $0.988,2.169$ \\
& $(0.072),(0.124)$ \\
MO-Fr( $(\alpha, a, b)$ & {$[0.85,1.13],[0.73,1.22]$} \\
& $131.615,2.141,1.419$ \\
GMO-Fr $(\theta, \alpha, a, b)$ & $(64.113),(0.171),(0.026)$ \\
\end{tabular}


(3.264), (4.002), (0.081), (29.314)

[16.19,28.99], [0,8.09], [0.22,0.53], [48.86,163.77]

$\mathrm{Kw}-\mathrm{Fr}(\beta, \delta, \mathrm{a}, \mathrm{b})$

4.607, 266.348, 0.278, 3.874

(1.541), (289.952), (0.053), (1.052)

[1.59,7.63], [0,834.65], [0.17,0.38], [1.81,5.94]

$\mathrm{B}-\operatorname{Fr}(\beta, \delta, \mathrm{a}, \mathrm{b})$

$\operatorname{KwMO}-\operatorname{Fr}(\alpha, \beta, \delta, \mathrm{a}, \mathrm{b})$

$0.672,40.928,0.445,47.781$

(0.364), (24.769), (0.092), (34.731)

[0,1.39], [0,89.42], [0.26,0.63], [0,115.85]

$0.502,2.636,57.290,0.304,22.304$

(0.461), (1.830), (53.645), (0.077), (39.976)

[0,1.41], [0,6.22], [0,162.43], [0.15,0.45], [0,100.65]

$\operatorname{MOKw}-\operatorname{Fr}(\alpha, \beta, \delta, \mathrm{a}, \mathrm{b})$

$110.479,1.944,22.595,0.3160 .975$

(186.517), (22.827), (47.590), (0.298), (6.093)

[0,476.05], [0,46.68], [0,115.87], [0,0.90], [0,12.92]

$\mathrm{XG}-\operatorname{Fr}(\theta, \mathrm{a}, \mathrm{b})$

$42.382,38.035,0.440$

(27.752), (33.957), (0.073)

[0,96.77], [0,104.59], [0.29,0.58]

Table 3: Log-likelihood, AIC, BIC, CAIC, HQIC, A, W and KS ( $p$-value) values for data set II.

\begin{tabular}{ccc}
\hline Models & AIC, BIC, CAIC, HQIC & A*, W*, K.S., and (p-value) \\
\hline Fr & $264.30,268.86,264.48,266.12$ & $4.76,0.78,0.22,(0.001)$ \\
MO-Fr & $219.21,226.04,219.56,221.93$ & $1.12,0.15,0.14,(0.10)$ \\
GMO-Fr & $214.94,224.06,215.53,218.54$ & $0.59,0.09,0.10,(0.48)$ \\
Kw-Fr & $216.14,225.24,216.73,219.76$ & $0.86,0.12,0.12,(0.29)$ \\
B-Fr & $219.52,228.63,220.12,223.15$ & $1.07,0.15,0.13,(0.16)$ \\
KwMO-Fr & $219.90,231.28,220.81,224.43$ & $0.95,0.13,0.12,(0.26)$ \\
MOKw-Fr & $210.84,222.22,211.75,215.37$ & $0.35,0.05,0.07,(0.81)$ \\
XG-Fr & $207.95,214.78,208.31,210.67$ & $0.28,0.03,0.06,(0.87)$ \\
\hline
\end{tabular}
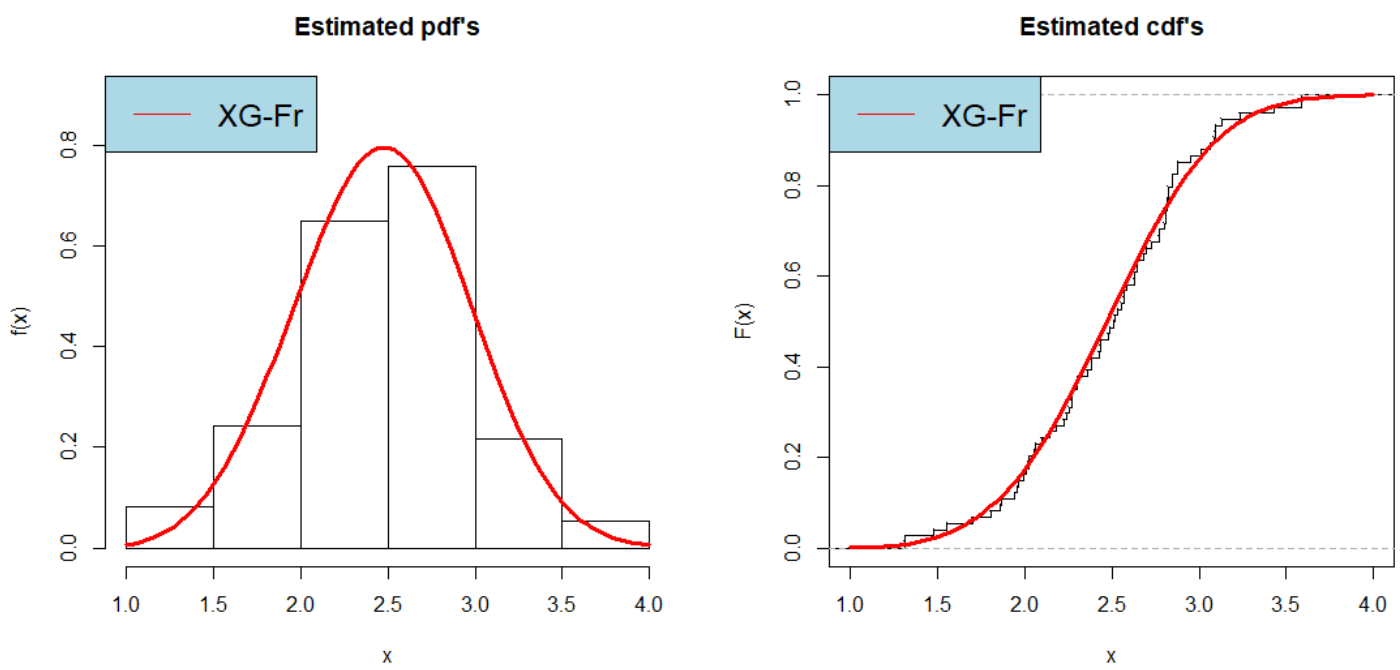
Figure 2: Estimated PDF and estimated CDF for data set I.
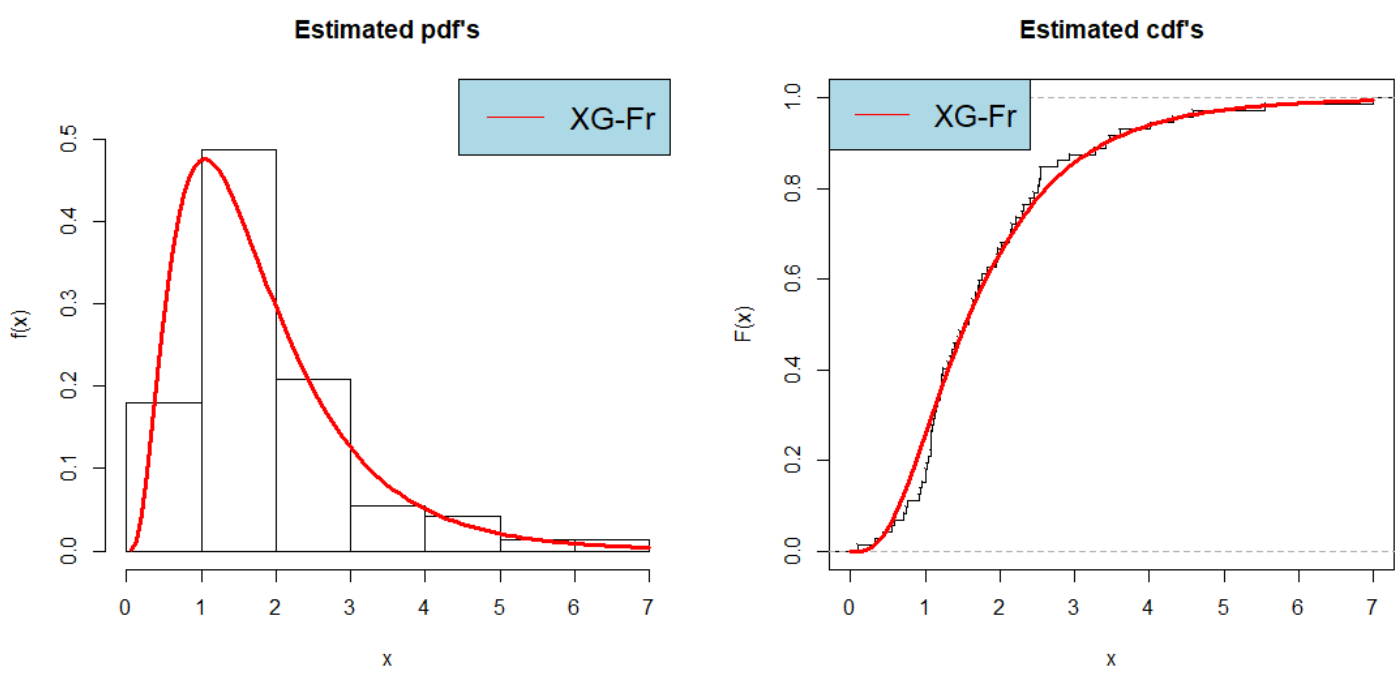

Figure 3: Estimated PDF and estimated CDF for data set II.

\section{Comparing methods}

\subsection{Using real data}

In this subsection, we consider the two real data sets to compare the classical methods for the purpose of comparison. We consider $\mathrm{W}^{*}$ and $\mathrm{A} *$ statistics.

Table 6: The values of estimators, $\mathrm{KS}, \mathrm{p}$-values, $\mathrm{W}^{*}$ and $\mathrm{A}^{*}$ for data I.

\begin{tabular}{cccccccc}
\hline Method & $\theta$ & $\mathrm{a}$ & $\mathrm{b}$ & $\mathrm{KS}$ & $\mathrm{p}$-value & $\mathrm{W}^{*}$ & $\mathrm{~A}^{*}$ \\
\hline ML & 78.5110 & 8.6620 & 1.229 & 0.05 & 0.98 & 0.04 & 0.29 \\
LS & 120.545 & 10.558 & 1.133 & 0.07 & 0.89 & 0.06 & 0.41 \\
WLS & 136.635 & 11.088 & 1.110 & 0.06 & 0.94 & 0.05 & 0.40 \\
CVM & 124.905 & 10.428 & 1.148 & 0.06 & 0.93 & 0.06 & 0.41 \\
Bootstrap. & 78.26400 & 8.6830 & 1.232 & 0.06 & 0.96 & 0.07 & 0.47 \\
\hline
\end{tabular}

From Table 6 we conclude that the ML method is the best method for modelling the data I. However, all other methods performed well.

Table 7: The values of estimators, KS, p-values, $\mathrm{W}^{*}$ and $\mathrm{A}^{*}$ for data II.

\begin{tabular}{cccccccc}
\hline Method & $\theta$ & $\mathrm{a}$ & $\mathrm{b}$ & $\mathrm{KS}$ & $\mathrm{p}$-value & $\mathrm{W}^{*}$ & $\mathrm{~A}^{*}$ \\
\hline ML & 22.583 & 12.815 & 0.567 & 0.112 & 0.324 & 0.103 & 0.743 \\
LS & 22.317 & 11.808 & 0.602 & 0.074 & 0.830 & 0.106 & 0.764 \\
WLS & 29.537 & 16.576 & 0.553 & 0.072 & 0.850 & 0.097 & 0.696 \\
CVM & 31.851 & 17.031 & 0.554 & 0.072 & 0.850 & 0.096 & 0.684 \\
Bootstrap. & 23.630 & 11.627 & 0.598 & 0.103 & 0.431 & 0.103 & 0.741 \\
\hline
\end{tabular}

From Table 7 we conclude that the CVM method is the best method for modelling the data II. However, all other methods performed well.

\subsection{Using simulated data}

In this subsection, a Monte Carlo simulation study is conducted for comparing the performance of the different estimators of the unknown parameters of the XG-Fr distribution. The performance of the different estimators proposed in the previous section is evaluated regarding their mean squared errors (MSEs). All the computations in this section are done by Mathcad program Version 15.0. We generate 1000 samples of the XG-Fr distribution for all:

$$
\theta, a, b=(0.8,0.5,2.0) \mid n=(20,50,100,200,500,100)
$$




$$
\theta, a, b=(2.0,1.5,6.0) \mid n=(20,50,100,200,500,100)
$$

The average values (AVs) of estimates and the corresponding MSEs of MLEs, LSEs, WLSEs, CVM, MPSD and Bootstrapping method are obtained and reported in Tables 8-13, we observe that all the estimates show the property of consistency, i.e., the MSEs decrease as sample size increase.

Table 8: Average values of estimates and the corresponding MSEs (in parentheses) for $n=20$.

\begin{tabular}{ccccccc}
\hline Parameters & MLE & LS & WLS & CVM & MPSD & Bootstrap \\
\hline$\theta=0.8$ & 0.82585 & 0.82003 & 0.82011 & 0.82907 & 0.87386 & 0.79067 \\
& $(0.02352)$ & $(0.02358)$ & $(0.02361)$ & $(0.02846)$ & $(0.04940)$ & $(0.02641)$ \\
& & & & & & \\
$\mathrm{a}=0.5$ & 0.52265 & 0.52971 & 0.53128 & 0.53092 & 0.48897 & 0.51165 \\
& $(0.01349)$ & $(0.02642)$ & $(0.02625)$ & $(0.10218)$ & $(0.011117)$ & $(0.00961)$ \\
& & & & & & \\
$\mathrm{b}=2$ & 1.91052 & 2.19642 & 2.20434 & 2.21164 & 1.63196 & 1.75413 \\
& $(0.11605)$ & $(0.87159)$ & $(0.94138)$ & $(0.76524)$ & $(0.23441)$ & $(0.16283)$ \\
& & & & & & \\
$\theta=2$ & 2.19316 & 2.02400 & 2.00874 & 2.04954 & 1.53874 & 2.76060 \\
& $(0.51443)$ & $(0.25803)$ & $(0.17793)$ & $(0.22906)$ & $(0.81699)$ & $(1.01390)$ \\
$\mathrm{a}=1.5$ & & & & & \\
& 1.73901 & 1.75595 & 1.80102 & 1.69294 & 1.35989 & 2.12633 \\
$\mathrm{~b}=0.6$ & $(0.62422)$ & $(1.09593)$ & $(1.15159)$ & $(0.73927)$ & $(0.33846)$ & $(0.76215)$ \\
& & & & & \\
& 0.61699 & 0.55183 & 0.69182 & 0.59682 & 0.54524 & 0.67957 \\
& $(0.01119)$ & $(0.76958)$ & $(0.38287)$ & $(0.07918)$ & $(0.01059)$ & $(0.01101)$ \\
\hline
\end{tabular}

Table 9: Average values of estimates and the corresponding MSEs (in parentheses) for $n=50$.

\begin{tabular}{ccccccc}
\hline Parameters & MLE & LS & WLS & CVM & MPSD & Bootstrap \\
\hline$\theta=0.8$ & 0.80575 & 0.81009 & 0.81088 & 0.80637 & 0.84157 & 0.81411 \\
& $(0.00747)$ & $(0.00946)$ & $(0.00919)$ & $(0.00836)$ & $(0.01593)$ & $(0.00897)$ \\
& & & & & & \\
$\mathrm{a}=0.5$ & 0.509831 & 0.51134 & 0.51128 & 0.51269 & 0.48744 & 0.50799 \\
& $(0.00408)$ & $(0.00824)$ & $(0.00738)$ & $(0.00736)$ & $(0.00383)$ & $(0.00487)$ \\
& & & & & & \\
$\mathrm{b}=2$ & 1.94043 & 2.07349 & 2.08209 & 2.05601 & 1.76653 & 1.93541 \\
& $(0.04942)$ & $(0.18704)$ & $(0.20593)$ & $(0.16037)$ & $(0.09891)$ & $(0.04770)$ \\
& & & & & & \\
$\theta=2$ & 2.08943 & 2.01674 & 2.00695 & 2.04475 & 1.73713 & 2.39516 \\
& $(0.16022)$ & $(0.08049)$ & $(0.06711)$ & $(0.08797)$ & $(0.30872)$ & $(0.40733)$ \\
$\mathrm{a}=1.5$ & & & & & \\
& 1.71343 & 1.56079 & 1.61719 & 1.53678 & 1.40121 & 1.74185 \\
$\mathrm{~b}=0.6$ & $(0.36012)$ & $(0.21760)$ & $(0.39769)$ & $(0.21074)$ & $(0.23317)$ & $(0.34283)$ \\
& & & & & \\
& 0.60925 & 0.58437 & 0.63833 & 0.60572 & 0.57117 & 0.63119 \\
& $(0.00372)$ & $(0.02898)$ & $(0.17867)$ & $(0.03796)$ & $(0.00387)$ & $(0.00463)$ \\
\hline
\end{tabular}

Table 10: Average values of estimates and the corresponding MSEs (in parentheses) for $n=100$.

\begin{tabular}{ccccccc}
\hline Parameters & MLE & LS & WLS & CVM & MPSD & Bootstrap \\
\hline$\theta=0.8$ & 0.80279 & 0.80289 & 0.80303 & 0.80228 & 0.82428 & 0.84302 \\
& $(0.00336)$ & $(0.00403)$ & $(0.00382)$ & $(0.00415)$ & $(0.00773)$ & $(0.00519)$ \\
& & & & & & \\
$\mathrm{a}=0.5$ & 0.50483 & 0.50665 & 0.50658 & 0.50755 & 0.49121 & 0.48934 \\
& $(0.00184)$ & $(0.00367)$ & $(0.00326)$ & $(0.00366)$ & $(0.00184)$ & $(0.00173)$
\end{tabular}




\begin{tabular}{ccccccc}
$\mathrm{b}=2$ & 1.96629 & 2.02497 & 2.02656 & 2.02356 & 1.82923 & 2.07014 \\
& $(0.02514)$ & $(0.06673)$ & $(0.06785)$ & $(0.06996)$ & $(0.05445)$ & $(0.02472)$ \\
& & & & & & \\
$\theta=2$ & 2.05281 & 2.00453 & 1.99416 & 2.01250 & 1.84775 & 1.90984 \\
& $(0.07132)$ & $(0.03206)$ & $(0.03064)$ & $(0.03267)$ & $(0.12547)$ & $(0.05779)$ \\
$\mathrm{a}=1.5$ & & & & & \\
& 1.65186 & 1.52825 & 1.55364 & 1.52721 & 1.41728 & 1.51985 \\
& $(0.19292)$ & $(0.09314)$ & $(0.11162)$ & $(0.10186)$ & $(0.14726)$ & $(0.10752)$ \\
$\mathrm{b}=0.6$ & 0.60599 & 0.58875 & 060169 & 0.59808 & 0.58214 & 0.58354 \\
& $(0.00167)$ & $(0.00382)$ & $(0.00823)$ & $(0.00429)$ & $(0.00179)$ & $(0.00166)$ \\
\hline
\end{tabular}

Table 11: Average values of estimates and the corresponding MSEs (in parentheses) for $n=200$.

\begin{tabular}{ccccccc}
\hline Parameters & MLE & LS & WLS & CVM & MPSD & Bootstrap \\
\hline$\theta=0.8$ & 0.80060 & 0.80331 & 0.80312 & 0.80123 & 0.81124 & 0.81681 \\
& $(0.00167)$ & $(0.00196)$ & $(0.00184)$ & $(0.00192)$ & $(0.00361)$ & $(0.00180)$ \\
& & & & & & \\
$\mathrm{a}=0.5$ & 0.50371 & 0.50139 & 0.50167 & 0.50329 & 0.49598 & 0.49827 \\
& $(0.00095)$ & $(0.00165)$ & $(0.00142)$ & $(0.00166)$ & $(0.00095)$ & $(0.00072)$ \\
& & & & & & \\
$\mathrm{b}=2$ & 1.98288 & 2.01913 & 2.01882 & 2.01139 & 1.88785 & 2.07138 \\
& $(0.01299)$ & $(0.03086)$ & $(0.03060)$ & $(0.03023)$ & $(0.02580)$ & $(0.01982)$ \\
& & & & & & \\
$\theta=2$ & 2.02419 & 1.99708 & 1.99421 & 2.00136 & 1.90102 & 1.95138 \\
& $(0.03476)$ & $(0.01389)$ & $(0.01316)$ & $(0.01512)$ & $(0.06678)$ & $(0.03280)$ \\
$\mathrm{a}=1.5$ & & & & & \\
& 1.59205 & 1.52077 & 1.52951 & 1.52176 & 1.43279 & 1.57543 \\
$\mathrm{~b}=0.6$ & $(0.10185)$ & $(0.04467)$ & $(0.04185)$ & $(0.04846)$ & $(0.10619)$ & $(0.08973)$ \\
& & & & & \\
& 0.60284 & 0.59422 & $0.60017)$ & 0.59701 & 0.58830 & 0.59315 \\
& $(0.00087)$ & $(0.00196)$ & $(0.00125$ & $(0.00201)$ & $(0.00103)$ & $(0.00091)$ \\
\hline
\end{tabular}

Table 12: Average values of estimates and the corresponding MSEs (in parentheses) for $\mathrm{n}=500$.

\begin{tabular}{|c|c|c|c|c|c|c|}
\hline Parameters & MLE & LS & WLS & CVM & MPSD & Bootstrap \\
\hline$\theta=0.8$ & $\begin{array}{c}0.80139 \\
(0.00069)\end{array}$ & $\begin{array}{c}0.79924 \\
(0.00075)\end{array}$ & $\begin{array}{c}0.79928 \\
(0.00070)\end{array}$ & $\begin{array}{c}0.80099 \\
(0.00079)\end{array}$ & $\begin{array}{c}0.80362 \\
(0.00125)\end{array}$ & $\begin{array}{c}0.79718 \\
(0.00067)\end{array}$ \\
\hline$a=0.5$ & $\begin{array}{c}0.50056 \\
(0.00038)\end{array}$ & $\begin{array}{c}0.50249 \\
(0.00067)\end{array}$ & $\begin{array}{c}0.50243 \\
(0.00057)\end{array}$ & $\begin{array}{c}0.50096 \\
(0.00069)\end{array}$ & $\begin{array}{c}0.49862 \\
(0.00034)\end{array}$ & $\begin{array}{c}0.49967 \\
(0.00037)\end{array}$ \\
\hline$b=2$ & $\begin{array}{c}1.99427 \\
(0.00559)\end{array}$ & $\begin{array}{c}1.99946 \\
(0.01128)\end{array}$ & $\begin{array}{c}1.99963 \\
(0.01101)\end{array}$ & $\begin{array}{c}2.00640 \\
(0.01185)\end{array}$ & $\begin{array}{c}1.94488 \\
(0.00876)\end{array}$ & $\begin{array}{c}1.96031 \\
(0.00560)\end{array}$ \\
\hline$\theta=2$ & $\begin{array}{c}2.00346 \\
(0.01345)\end{array}$ & $\begin{array}{c}1.99548 \\
(0.00547)\end{array}$ & $\begin{array}{c}1.99820 \\
(0.00501)\end{array}$ & $\begin{array}{c}2.00267 \\
(0.00570)\end{array}$ & $\begin{array}{c}1.96099 \\
(0.02201)\end{array}$ & $\begin{array}{c}1.94357 \\
(0.01556)\end{array}$ \\
\hline$a=1.5$ & $\begin{array}{c}1.53029 \\
(0.03029)\end{array}$ & $\begin{array}{c}1.51408 \\
(0.01774)\end{array}$ & $\begin{array}{c}1.50817 \\
(0.01373)\end{array}$ & $\begin{array}{c}1.50441 \\
(0.01801)\end{array}$ & $\begin{array}{c}1.46717 \\
(0.04788)\end{array}$ & $\begin{array}{c}1.44680 \\
(0.03429)\end{array}$ \\
\hline$b=0.6$ & $\begin{array}{l}0.600153 \\
(0.00034)\end{array}$ & $\begin{array}{c}0.59686 \\
(0.00082)\end{array}$ & $\begin{array}{l}0.600062 \\
(0.00048)\end{array}$ & $\begin{array}{c}0.60027 \\
(0.00086)\end{array}$ & $\begin{array}{c}0.59526 \\
(0.00035)\end{array}$ & $\begin{array}{c}0.59084 \\
(0.00041)\end{array}$ \\
\hline
\end{tabular}


Table 13: Average values of estimates and the corresponding MSEs (in parentheses) for $n=1000$.

\begin{tabular}{ccccccc}
\hline Parameters & MLE & LS & WLS & CVM & MPSD & Bootstrap \\
\hline$\theta=0.8$ & 0.80002 & 0.79951 & 0.79945 & 0.79946 & 0.80199 & 0.79414 \\
& $(0.00033)$ & $(0.00038)$ & $(0.00034)$ & $(0.00037)$ & $(0.00062)$ & $(0.00036)$ \\
$\mathrm{a}=0.5$ & 0.50045 & 0.50135 & 0.50137 & 0.50139 & 0.49916 & 0.50345 \\
& $(0.00017)$ & $(0.00033)$ & $(0.00027)$ & $(0.00032)$ & $(0.00017)$ & $(0.00020)$ \\
& & & & & & \\
$\mathrm{b}=2$ & 1.99402 & 1.99934 & 1.99907 & 1.99912 & 1.96705 & 1.97195 \\
& $(0.00263)$ & $(0.00564)$ & $(0.00534)$ & $(0.00549)$ & $(0.00364)$ & $(0.00319)$ \\
& & & & & & \\
$\theta=2$ & 2.00525 & 1.99790 & 1.99847 & 1.99891 & 1.97613 & 1.96653 \\
& $(0.00648)$ & $(0.00265)$ & $(0.00244)$ & $(0.00265)$ & $(0.01059)$ & $(0.00748)$ \\
$\mathrm{a}=1.5$ & & & & & \\
& 1.52481 & 1.50723 & 1.50782 & 1.50662 & 1.47577 & 1.47828 \\
$\mathrm{~b}=0.6$ & $(0.02016)$ & $(0.00835)$ & $(0.00662)$ & $(0.00832)$ & $(0.02747)$ & $(0.01780)$ \\
& & & & & \\
& 0.60079 & 0.59880 & 0.60034 & 0.599613 & 0.59715 & 0.59504 \\
& $(0.00017)$ & $(0.00040)$ & $(0.00023)$ & $(0.00040)$ & $(0.00017)$ & $(0.00019)$ \\
\hline
\end{tabular}

\section{Concluding remarks}

A new extension of the Fréchet model called the Xgamma Fréchet model is proposed. Some of its properties such as ordinary moments, incomplete moments and moment generating function are derived. The estimation of the parameters is carried out via different methods. The performances of the proposed estimation methods are studied through Monte Carlo simulations and real-life data sets. The potentiality of the proposed model is analyzed through modelling of two real life data sets. As a future work, we can use and apply many new goodness-of-fit statistic tests for right censored distributional validation such as the "Nikulin-Rao-Robson goodness-of-fit statistical test", the "modified Nikulin-Rao-Robson goodness-of-fit statistical test", the "Bagdonavicius-Nikulin goodness-of-fit statistical test" and the "modified Bagdonavicius-Nikulin goodness-of-fit statistical test" as performed by Ibrahim et al. (2019 and 2020a,b), Goual et al. (2019, 2020), Mansour et al. (2020a,b,c,d), Yadav et al. (2020) and Goual and Yousof (2020), among others. However, many types of copulas can be used and applied for deriving many new bivariate models based on the new distribution (see Al-babtain et al. (2020a), Elgohari and Yousof (2020a,b), Mansour et al. (2020e, f), Ali et al. (2021a,b) and El-Morshedy et al. (2021) for more details).

\section{References}

1. Al-babtain, A. A., Elbatal, I. and Yousof, H. M. (2020a). A New Flexible Three-Parameter Model: Properties, Clayton Copula, and Modeling Real Data. Symmetry, 12(3), 440.

2. Al-Babtain, A. A., Elbatal, I. and Yousof, H. M. (2020b). A new three parameter Fréchet model with mathematical properties and applications. Journal of Taibah University for Science, 14(1), 265-278.

3. Ali, M. M., Yousof, H. M. and Ibrahim, M. A New Version of the Generalized Rayleigh Distribution with Copula, Properties, Applications and Different Methods of Estimation. (2021a) Optimal Decision Making in Operations Research \& Statistics: Methodologies and Applications, CRC Press, To appear.

4. Ali, M. M., Ibrahim, M. and Yousof, H. M. (2021b). Expanding the Burr X Model: Properties, Copula, Real Data Modeling and Different Methods of Estimation. Optimal Decision Making in Operations Research \& Statistics: Methodologies and Applications, CRC Press, To appear.

5. Alizadeh, M., Jamal, F., Yousof, H. M., Khanahmadi, M. and Hamedani, G. G. (2020). Flexible Weibull generated family of distributions: characterizations, mathematical properties and applications. University Politehnica of Bucharest Scientific Bulletin-Series A-Applied Mathematics and Physics, 82(1), 145-150.

6. Aryal, G. R. and Yousof, H. M. (2017). The exponentiated generalized-G Poisson family of distributions. Stochastics and Quality Control, 32(1), 7-23.

7. Aryal, G. R., Ortega, E. M., Hamedani, G. G. and Yousof, H. M. (2017). The Topp-Leone generated Weibull distribution: regression model, characterizations and applications. International Journal of Statistics and Probability, 6(1), 126-141. 
8. Barreto-Souza, W. M., Cordeiro, G. M. and Simas, A. B. (2011). Some results for beta Fréchet distribution. Communication in Statistics-Theory and Methods, 40, 798-811.

9. Bjerkedal, T. (1960). Acquisition of resistance in Guinea pigs infected with different doses of virulent tubercle bacilli. American Journal of Hygiene, 72, 130-148.

10. Brito, E., Cordeiro, G. M., Yousof, H. M., Alizadeh, M. and Silva, G. O. (2017). The Topp-Leone odd log-logistic family of distributions. Journal of Statistical Computation and Simulation, 87(15), 3040-3058.

11. Chakraborty, S., Handique, L., Altun, E. and Yousof, H. M. (2018). A new statistical model for extreme values: mathematical properties and applications. International Journal of Open Problems in Computer Science and Mathematics, 12(1), 1-18.

12. Cheng, R. C. H. and Amin, N. A. K. (1983). Estimating parameters in continuous univariate distributions with a shifted origin. J. R. Statist. Soc. B, 45(3), 394-403.

13. Cordeiro, G. M., Altun, E., Korkmaz, M. C., Pescim, R. R. and Afify, A. Z. and Yousof, H. M. (2020). The xgamma Family: Censored Regression Modelling and Applications. Revstat Statistical Journal, 18(5), 593-612.

14. Cordeiro, G. M., Yousof, H. M., Ramires, T. G. and Ortega, E. M. (2018). The Burr XII system of densities: properties, regression model and applications. Journal of Statistical Computation and Simulation, 88(3), $432-456$.

15. Elgohari, H. and Yousof, H. M. (2020a). A Generalization of Lomax Distribution with Properties, Copula and Real Data Applications. Pakistan Journal of Statistics and Operation Research, 16(4), 697-711.

16. Elgohari, H. and Yousof, H. M. (2020b). New Extension of Weibull Distribution: Copula, Mathematical Properties and Data Modeling. Statistics, Optimization \& Information Computing, 8(4), 972-993. https://doi.org/10.19139/soic-2310-5070-1036.

17. El-Morshedy, M., Alshammari, F. S., Hamed, Y. S., Eliwa, M. S., Yousof, H. M. (2021). A New Family of Continuous Probability Distributions. Entropy, 23, 194.

18. Elsayed, H. A. and Yousof, H. M. (2019a). A new Lomax distribution for modeling survival times and taxes revenue data sets. Journal of Statistics and Applications, forthcoming.

19. Elsayed, H. A. H. and Yousof, H. M. (2019b). The Burr X Nadarajah Haghighi distribution: statistical properties and application to the exceedances of flood peaks data. Journal of Mathematics and Statistics, 15, 146-157.

20. Elsayed, H. A. H. and Yousof, H. M. (2020). The generalized odd generalized exponential Fréchet model: univariate, bivariate and multivariate extensions with properties and applications to the univariate version. Pakistan Journal of Statistics and Operation Research, 529-544.

21. Gad, A. M., Hamedani, G. G., Salehabadi, S. M. and Yousof, H. M. (2019). The Burr XII-Burr XII distribution: mathematical properties and characterizations. Pakistan Journal of Statistics, 35(3), 229-248.

22. Goual, H., Yousof, H. M. and Ali, M. M. (2019). Validation of the odd Lindley exponentiated exponential by a modified goodness of fit test with applications to censored and complete data. Pakistan Journal of Statistics and Operation Research, 15(3), 745-771.

23. Goual, H. and Yousof, H. M. (2020). Validation of Burr XII inverse Rayleigh model via a modified chi-squared goodness-of-fit test. Journal of Applied Statistics, 47(3), 393-423.

24. Goual, H., Yousof, H. M. and Ali, M. M. (2020). Lomax inverse Weibull model: properties, applications, and a modified Chi-squared goodness-of-fit test for validation. Journal of Nonlinear Sciences \& Applications (JNSA), 13(6), 330-353.

25. Gradshteyn, I.S. and Ryzhik, I.M. (2002). Table of integrals, series, and products. San Diego, CA: Academic Press.

26. Ibrahim, M., Altun, E., Goual, H., and Yousof, H. M. (2020a). Modified goodness-of-fit type test for censored validation under a new Burr type XII distribution with different methods of estimation and regression modeling. Eurasian Bulletin of Mathematics, 3(3), 162-182.

27. Ibrahim, M., Altun, E. and Yousof, H. M. (2020b). A new distribution for modeling lifetime data with different methods of estimation and censored regression modeling. Statistics, Optimization \& Information Computing, $8(2), 610-630$.

28. Ibrahim, M., Yadav, A. S., Yousof, H. M., Goual, H. and Hamedani, G. G. (2019). A new extension of Lindley distribution: modified validation test, characterizations and different methods of estimation. Communications for Statistical Applications and Methods, 26(5), 473-495.

29. Jahanshahi, S. M. A., Yousof, H. M. and Sharma, V. K. (2019). The Burr X Fréchet Model for Extreme Values: Mathematical Properties, Classical Inference and Bayesian Analysis. Pak. J. Stat. Oper. Res., 15(3), $797-818$.

30. Karamikabir, H., Afshari, M., Yousof, H. M., Alizadeh, M. and Hamedani, G. (2020). The Weibull Topp-Leone Generated Family of Distributions: Statistical Properties and Applications. Journal of The Iranian Statistical Society, 19(1), 121-161. 
31. Korkmaz, M. C., Yousof, H. M. and Ali, M. M. (2017). Some theoretical and computational aspects of the odd Lindley Fréchet distribution. İstatistikçiler Dergisi: İstatistik ve Aktüerya, 10(2), 129-140.

32. Korkmaz, M. C., Yousof, H. M., Hamedani G. G. and Ali, M. M. (2018). The Marshall--Olkin generalized G Poisson family of distributions, Pakistan Journal of Statistics, 34, 251-267.

33. Lak, F., Basikhasteh, M., Alizadeh, M. and Yousof, H. M. (2019). The Odd Log-Logistic Generalized HalfNormal Lifetime Poisson Model. Pakistan Journal of Statistics and Operation Research, 15(1), 111-128.

34. Harlow, D. G. (2002). Applications of the Fréchet distribution function, International Journal of Materials and Product Technology, 17, 482-495.

35. Johnson, N. L., Kemp, A. W. and Kotz, S. (2005). Univariate discrete distributions, 3rd edn. Wiley, Hoboken.

36. Krishna, E., Jose, K. K., Alice, T. and Risti, M. M. (2013). The Marshall-Olkin Fréchet distribution. Communications in Statistics-Theory and Methods, 42, 4091- 4107.

37. Kundu, D. and Raqab, M. Z. (2009). Estimation of $\mathrm{R}=\mathrm{P}(\mathrm{Y}<\mathrm{X})$ for three parameter Weibull distribution. Statistics and Probability Letters, 79, 1839-1846.

38. Mansour, M. M., Ibrahim, M., Aidi, K., Shafique Butt, N., Ali, M. M., Yousof, H. M. and Hamed, M. S. (2020a). A New Log-Logistic Lifetime Model with Mathematical Properties, Copula, Modified Goodness-of-Fit Test for Validation and Real Data Modeling. Mathematics, 8(9), 1508.

39. Mansour, M. M., Butt, N. S., Ansari, S. I., Yousof, H. M., Ali, M. M. and Ibrahim, M. (2020b). A new exponentiated Weibull distribution's extension: copula, mathematical properties and applications. Contributions to Mathematics, 1 (2020) 57-66. DOI: 10.47443/cm.2020.0018.

40. Mansour, M., Korkmaz, M. Ç., Ali, M. M., Yousof, H. M., Ansari, S. I. and Ibrahim, M. (2020c). A generalization of the exponentiated Weibull model with properties, Copula and application. Eurasian Bulletin of Mathematics, $3(2), 84-102$.

41. Mansour, M., Rasekhi, M., Ibrahim, M., Aidi, K., Yousof, H. M. and Elrazik, E. A. (2020d). A New Parametric Life Distribution with Modified Bagdonavičius-Nikulin Goodness-of-Fit Test for Censored Validation, Properties, Applications, and Different Estimation Methods. Entropy, 22(5), 592.

42. Mansour, M., Yousof, H. M., Shehata, W. A. and Ibrahim, M. (2020e). A new two parameter Burr XII distribution: properties, copula, different estimation methods and modeling acute bone cancer data. Journal of Nonlinear Science and Applications, 13 (5), 223-238.

43. Mansour, M. M., Butt, N. S., Yousof, H. M., Ansari, S. I. and Ibrahim, M. (2020f). A Generalization of Reciprocal Exponential Model: Clayton Copula, Statistical Properties and Modeling Skewed and Symmetric Real Data Sets. Pakistan Journal of Statistics and Operation Research, 16(2), 373-386.

44. Salah, M. M., El-Morshedy, M., Eliwa, M. S. and Yousof, H. M. (2020). Expanded Fréchet Model: Mathematical Properties, Copula, Different Estimation Methods, Applications and Validation Testing. Mathematics, 8(11), 1949.

45. Silva, R., de Andrade, T. A., Maciel, D., Campos, R. P. S., and Cordeiro, G. M. (2013). A new lifetime model: The gamma extended Fréchet distribution. Journal of Statistical Theory and Applications, 12, 39-54.

46. Swain, J. Venkatraman, S. Wilson, J. (1988). Least squares estimation of distribution function in Johnsons translation system, J. Stat. Comput. Simul., 29, 271-297.

47. Wright, E. M. (1935). The asymptotic expansion of the generalized hypergeometric function. Journal of the London Mathematical Society, 10, 286-293.

48. ul Haq, M. A., Yousof, H. M. and Hashmi, S. (2017). A New Five-Parameter Fréchet Model for Extreme Values. Pakistan Journal of Statistics and Operation Research, 617-632.

49. Yadav, A. S., Altun, E. and Yousof, H. M. (2019). Burr-Hatke Exponential Distribution: A Decreasing Failure Rate Model, Statistical Inference and Applications. Annals of Data Science, 1-20.

50. Yadav, A. S., Goual, H., Alotaibi, R. M., Ali, M. M. and Yousof, H. M. (2020). Validation of the Topp-LeoneLomax model via a modified Nikulin-Rao-Robson goodness-of-fit test with different methods of estimation. Symmetry, 12(1), 57.

51. Yousof, H. M., Afify, A. Z., Alizadeh, M., Butt, N. S., Hamedani, G. G. and Ali, M.M. (2015). The transmuted exponentiated generalized-G family of distributions. Pakistan Journal of Statistics and Operation Research, 11, 441-464.

52. Yousof, H. M., Afify, A. Z., Ebraheim, A. N., Hamedani, G. G. and Butt, N. S. (2016). On six-parameter Fréchet distribution: properties and applications, Pakistan Journal of Statistics and Operation Research 12, 281-299.

53. Yousof, H. M., Alizadeh, M., Jahanshahiand, S. M. A., Ramires, T. G., Ghosh, I. and Hamedani G. G. (2017a). The transmuted Topp-Leone $\mathrm{G}$ family of distributions: theory, characterizations and applications, Journal of Data Science. 15, 723-740. 
54. Yousof, H. M., Altun, E. and Hamedani, G. G. (2018a). A new extension of Frechet distribution with regression models, residual analysis and characterizations. Journal of Data Science, 16(4), 743-770.

55. Yousof, H. M., Altun, E., Rasekhi, M., Alizadeh, M., Hamedani, G. G. and Ali, M. M. (2019). A new lifetime model with regression models, characterizations and applications. Communications in Statistics-Simulation and Computation, 48(1), 264-286.

56. Yousof, H. M., Alizadeh, M., Jahanshahiand, S. M. A., Ramires, T. G., Ghosh, I. and Hamedani G. G. (2017b). The transmuted Topp-Leone $\mathrm{G}$ family of distributions: theory, characterizations and applications, Journal of Data Science. 15, 723-740.

57. Yousof, H. M., Hamedani, G. G., \& Ibrahim, M. (2020). The Two-parameter Xgamma Fréchet Distribution: Characterizations, Copulas, Mathematical Properties and Different Classical Estimation Methods. Contributions to Mathematics, 2 (2020), 32-41.

58. Yousof, H. M., Jahanshahi, S. M., Ramires, T. G Aryal, G. R. and Hamedani G. G. (2017c). A new distribution for extreme values: regression model, characterizations and applications. Journal of Data Science, Journal of Data Science, 16(4), 677-706.

59. Yousof, H. M., Majumder, M., Jahanshahi, S. M. A., Masoom Ali, M. and Hamedani, G. G. (2018b). A new Weibull class of distributions: theory, characterizations and applications. Journal of Statistical Research of Iran JSRI, 15(1), 45-82.

60. Yousof, H. M., Rasekhi, M., Altun, E. and Alizadeh, M. (2018c). The extended odd Fréchet family of distributions: properties, applications and regression modeling. International Journal of Applied Mathematics and Statistics, 30(1), 1-30.

61. Zaharim, A., Najid, S.K., Razali, A.M. and Sopian, K. (2009). Analyzing Malaysian wind speed data using statistical distribution, Proceedings of the 4th IASME/WSEAS International Conference on Energy and Environment, Cambridge. 